\title{
A NOTAÇÃO LOGARÍTMICA COMO OPÇÃO DE REGISTRO OBJETIVO DA ESTESIOMETRIA: UM ESTUDO PILOTO
}

\author{
THE LOGARITHMIC NUMBER AS AN OPTION OF OBJECTIVE REGISTRATION \\ OF ESTESIOMETRY: A PILOT STUDY
}

\section{Priscila da Silva Azevedo ${ }^{1}$ \\ Silvania Morais Cavalcante ${ }^{2}$ \\ Thamyres Pereira Alves ${ }^{3}$ \\ Gabriela Ferraz Jaime ${ }^{4}$ \\ Ananda Quaresma Nascimento ${ }^{5}$ \\ Marcelo Marques Cardoso 6}

Submissão à Plataforma Brasil (CAAE: 45352815.3.0000.0018) e aprovado por Comitê de Ética e Pesquisa, parecer n ${ }^{\circ}$ 1.337. 714.

\section{Autor Correspondente}

Prof. Dr. Marcelo Marques Cardoso

Universidade Federal do Pará.

Rua Augusto Corrêa, 01 - Guamá/ Belém - Pará. CEP.: 66075-110. Complemento: Faculdade de Fisioterapia e Terapia Ocupacional/ ICS UFPA.

(91) 98384-3048 (WhatsApp)

marcelomc@ufpa.br

${ }^{1}$ Mestranda Programa de Pós-Graduação em Ciências do Movimento Humano/ Instituto de Ciências da Saúde - Universidade Federal do Pará, (Belém, Pará - Brasil).

azevedoopriscila@gmail.com

${ }^{2}$ Graduada Faculdade de Fisioterapia e Terapia Ocupacional/ Instituto de Ciências da Saúde - Universidade Federal do Pará, (Belém, Pará Brasil).

silvania_morais95@hotmail.com

${ }^{3}$ Graduada Faculdade de Fisioterapia e Terapia Ocupacional/ Instituto de Ciências da Saúde - Universidade Federal do Pará, (Belém, Pará Brasil).

thamyresalves36@gmail.com

${ }^{4}$ Graduada. Faculdade de Fisioterapia e Terapia Ocupacional/ Instituto de Ciências da Saúde - Universidade Federal do Pará, (Belém, Pará Brasil).

gabrielajaime69@gmail.com

${ }^{5}$ Residência em Oncologia/ Hospital Universitário João de Barros Barreto - Universidade Federal do Pará, (Belém, Pará - Brasil). anandanascimento@yahoo.com.br

${ }^{6}$ Doutor do Laboratório de NeuroPlasticidade, Faculdade de Fisioterapia e Terapia Ocupacional/ Instituto de Ciências da Saúde Universidade Federal do Pará, (Belém, Pará - Brasil). marcelomc@ufpa.br

\section{Cite como \\ Vancouver}

\section{Resumo}

Introdução: A Avaliação objetiva é um valioso recurso na pesquisa. Contudo, o estesiômetro provê informações por cores.

Objetivo: Apresentar a conversão logarítmica dos valores nominais do estesiômetro como opção representativa de registro na avaliação de prejuízo tátil.

Método: Estudo Piloto aprovado com parecer n ${ }^{\circ} 1.337$. 714, realizado de agosto de 2018 a julho de 2019. Valores nominais $(\mathrm{gF})$ dos monofilamentos do estesiômetro foram convertidos em logaritmo, o procedimento serviu para o registro e o acompanhamento de pacientes cerebrovasculares em reabilitação $(n=13)$, comparado ao controle $(n=10)$, para notações de qualidade, acurácia e poder de inferências dos dados na representação do estado tátil. Statistical analysis performed by the GraphPad Prism 6.0 Program.

Resultados: As opções numéricas trabalhadas conseguem representar a percepção da sensibilidade tátil, sendo que a conversão em logaritmo demonstra objetividade, sensibilidade, coerência e consistência para gerar mais inferências.

Conclusão: A notação logarítmica para registro da sensibilidade é uma opção versátil de análise e interpretação dos dados na pesquisa em reabilitação.

Descritores: Percepção tátil. Avaliação da deficiência. Reabilitação. Fisioterapia. Terapia Ocupacional.

\begin{abstract}
Introduction: An Objective evaluation is a valuable resource in research. However, the information on the esthesiometer is by color.

Objective: To present the logarithmic conversion of the nominal values of the esthesiometer as a representative recording option in the assessment of tactile injury.

Method: Pilot study approved by the Ethics and Research Committee, technical opinion No. 1,337.714, carried out from August 2018 to July 2019. Nominal values (gF) of the colors of the monofilament were converted into a logarithm, the procedure was used to record and monitor cerebrovascular patients undergoing rehabilitation $(n=13)$, compared to control $(n=10)$, for quality ratings, accuracy and power of inferences of the data in the representation of the tactile state. Statistical analysis performed by the GraphPad Prism 6.0 Program.

Results: The numerical options operated managed to represent the tactile perception and the conversion to logarithm demonstrates objectivity, sensitivity, coherence and consistency to generate more inferences.

Conclusion: Logarithmic notation for recording sensitivity is a versatile option for analyzing and interpreting data in rehabilitation research.
\end{abstract}

Keywords: Touch perception. Disability evaluation. Rehabilitation. Physiotherapy. Occupational Therapy.

Azevedo, PS, Cavalcante, SM, Alves, TP, Jaime, GF, Nascimento, AQ, Cardoso, MM. A notação logarítmica como opção de registro objetivo da estesiometria: um estudo piloto. Conscientiae Saúde 2020;19(1):1-15, e17642. https://doi.org/10.5585/conssaude.v19n1.17642. 


\section{Introdução}

Testes físicos são importantes instrumentos para avaliação e acompanhamento de pacientes em reabilitação e servem para o registro psicométrico do estado físico funcional prejudicados por diferentes acometimentos sensoriomotores ${ }^{1}$, em particular, prejuízos perceptivos da sensibilidade tátil são recorrentes sequelas neurológicas, como as causadas por um Acidente Vascular Encefálico $(\mathrm{AVE})^{2}$. Na contramão desse entendimento, é notório na literatura como as sequelas motoras recebem maior importância no AVE, em detrimento aos estudos focados no peso que os danos somatossensoriais têm sobre a recuperação funcional geral e à independência nas Atividades da Vida Diária dos pacientes ${ }^{3}$. Isso pode ser uma causa de desequilíbrio na qualidade entre instrumentos de avaliação física, sensitiva e motora. Para a coleta destas e outras variáveis são utilizados instrumentos e testes, analógicos e digitais que auxiliam na interpretação da resposta dos pacientes por um determinado conjunto de procedimentos terapêuticos ${ }^{4}$, sejam eles físicos, ocupacionais e fisiátricos. Entre os quais se destacam o dinamômetro, o goniômetro e o estesiômetro ${ }^{5}$.

O uso desses e outros testes físicos na pesquisa em reabilitação prima pela métrica e reprodutividade para gerar inferências sustentadas na precisão e na qualidade dos dados ${ }^{1,5}$. Os dispositivos analógicos, mesmo os validados, usualmente manifestam poder de reprodutividade com maiores desvios que seus paralelos digitais ${ }^{6,7}$, como é o caso do estesiômetro do tipo monofilamentos de Semmes-Weinstein ${ }^{8}$. Em parte, o comprometimento da acurácia dos dados durante a coleta é causado por variabilidade entre avaliadores diferentes ou pela dispersão entre as áreas das secções transversais de um monofilamento para outro, com valores nominais notadamente diferentes ${ }^{9}$. Isso pode ser um problema quando o monofilamento em teste tem secção transversal maior que o necessário para estimular o campo receptivo da pele ou o atual estado de sensibilidade. Sendo assim, seria mais compatível com um monofilamento mais delicado ao passo que a resposta emerge por outro, mais calibroso. Essas ocorrências ocultam a percepção sensitiva tátil real do sujeito em função do registro qualitativo pela cor do estesiômetro usado ${ }^{10}$. Esses exemplos, são de fato difíceis de serem corrigidos em um conjunto de estesiômetro com poucos componentes, como é o caso do represente mais utilizado no País, com seis monofilamentos, frente a similares com mais monofilamentos no conjunto, como o estesiômetro de von Frey. Assim, emergiu o interesse de aplicação do teste de sensibilidade tátil, com o estesiômetro nacional, baseado em parâmetros objetivos - convertendo o caráter descritivo qualitativo das cores para valores numéricos congruentes. 
Disso, surge a questão de qual notação numérica usar? Uma vez que os valores nominais dos monofilamentos do kit mais utilizado no Brasil, em grama-força ( $\mathrm{gF}$ ), parecem bem discrepantes entre si, comparado à possibilidade de harmonia da notação logarítmica. Pelo exposto, objetiva-se identificar a opção mais representativa, viável e reprodutível de registro numérico de avaliações mediadas pelos monofilamentos do estesiômetro, se por valores em grama-força $(\mathrm{gF})$ ou se convertidos em logaritmo de base $10(\log 10)$, em resposta ao tratamento de reabilitação de pacientes com a percepção sensitiva prejudicada.

\section{Materiais e métodos}

\subsection{Apresentação geral e aprovação}

Estudo piloto realizado de agosto de 2018 a julho de 2019 com 23 participantes, ordenados em grupos de controle e intervenção, em ambulatório escola de reabilitação física de uma Instituição Federal de Ensino Superior. Submissão à Plataforma Brasil (CAAE: 45352815.3.0000.0018) e aprovado por Comitê de Ética e Pesquisa, parecer n ${ }^{\circ} 1.337 .714$.

Os critérios de seleção abrangeram pacientes com diagnóstico clínico de Acidente Vascular Encefálico (AVE) isquêmico ou hemorrágico, maiores de 18 anos, com pelo menos 06 meses da ocorrência e alta de serviços de reabilitação por mais de 03 meses. Os controles foram sujeitos saudáveis que seguiram os critérios demográficos semelhantes aos sobreviventes de AVE. Todos os selecionados realizaram previamente o teste de rastreio cognitivo, pelo Mini Exame do Estado Mental (MEEM), para garantir que os sujeitos teriam condições ideais de compreensão dos comandos e dos procedimentos realizados ${ }^{11}$.

\subsection{Procedimentos e formação dos grupos}

Dados dos testes táteis de pacientes selecionados de um projeto de reabilitação, baseados no treinamento de Atividade da Vida Diária (AVDs), com complementação de Gamificação e Prática Mental, foram editados para averiguar a eficácia do tratamento de sequelas do AVE, totalizaram 23 participantes assim agrupados: Grupo Controle Saudável (GCS), n=10 voluntários com a sensibilidade tátil preservada; e Grupo de Tratamento AVE (GTA), n=13 pacientes incluídos para reabilitação. O fluxograma resume os procedimentos adotados (Fig. $01)$. 
Figura 01 - Fluxograma. Apresentação geral dos projetos e reagrupamento dos participantes

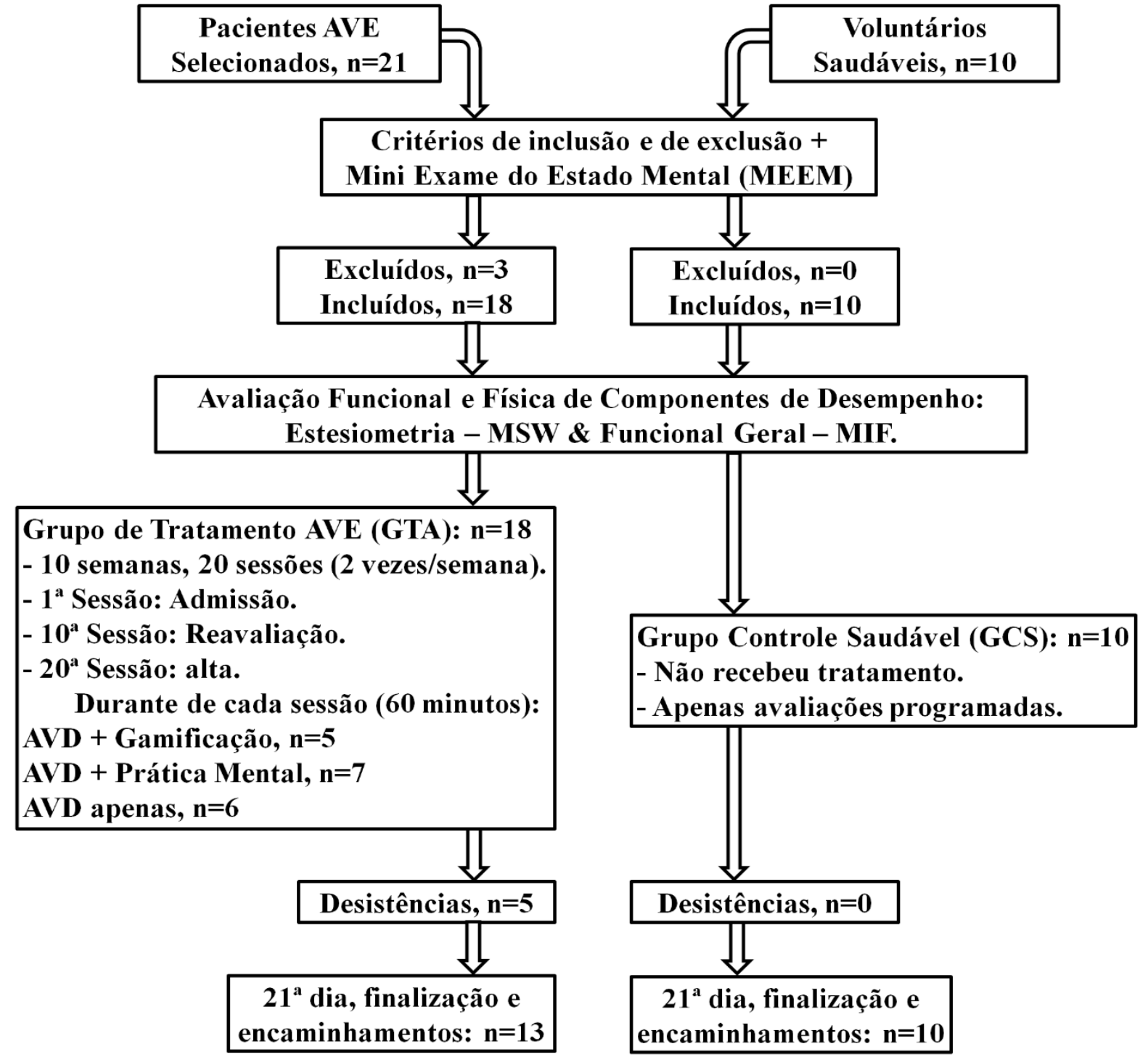

Legenda: Medida de Independência Funcional (MIF). Graduação da Força Muscular pela Escala de Medical Research Council (MRC). Monofilamento de Semmes-Weinstein (MSW).

Fonte: Própria da autoria.

\subsection{Avaliação funcional e estesiometria}

A recuperação em resposta à intervenção geral foi analisada pela Medida de Independência Funcional (MIF), uma escala de 18 itens, agrupadas em seis domínios ${ }^{12}$. Cada item é pontuado de 1 a 7 , correspondendo, respectivamente, à dependência total e à independência completa, assim a pontuação total varia de 18 a 126 pontos ${ }^{13}$. Contudo, na admissão, os itens que registraram 7 pontos foram integralmente retirados da análise dos dados, considerando-se apenas o acompanhamento subsequente dos itens com valores iguais ou menores a 6 pontos, sem os somar ao final. Brevemente: Esse procedimento garantiu a análise do estado evolutivo de itens dos domínios da MIF que estavam com algum grau de prejuízo, 
sem a dissolução do estado funcional geral proveniente da soma com os itens com notação inicial de independência completa (7 pontos). Assim, nos três tempos de avaliação, ordenou-se a média apenas dos itens considerados na admissão até a alta de cada paciente para gerar a análise evolutiva do grupo. Esta notação foi eficiente no momento de prover a correlação com os achados da estesiometria.

O kit do estesiômetro $\left(\right.$ Sorri-Bauru $\left.{ }^{\circledR}\right)$ foi usado no teste tátil ${ }^{14}$. Resumidamente: o conjunto contém seis monofilamentos de Semmes-Weinstein (MSW), com área de secção transversal e valores nominais específicos (Quadro 01). Os procedimentos de coleta seguiram integralmente o manual do produto e foram realizados pelo mesmo pesquisador nas três janelas temporais de checagem ${ }^{15}$. Os momentos de checagem foram usados apenas para realizar os testes físico e Funcional (Fig. 01). Todos os procedimentos foram repetidos três vezes pelo pesquisador e registrados com descanso de 5 minutos entre uma tentativa e outra.

\subsection{Conversão em logaritmo de base 10}

O quadro 1 resume o procedimento matemático aplicado para a conversão em logaritmo de base 10 (Log10). Brevemente: o valor nominal de cada monofilamento $(\mathrm{gF})$ foi multiplicado por 100 (para se evitar valores de Log 10 negativos) ${ }^{16}$. Os resultados encontrados foram usados como correspondentes numéricos à descrição qualitativa das cores dos monofilamentos ${ }^{15}$.

Durante as coletas, convencionou-se representar a falta de percepção tátil a todos os monofilamentos, pelo registro da cor preta no ponto testado. Para esta cor foi padronizada 900 (gF) como valor nominal de referência - três vezes o valor nominal do estesiômetro rosa (quadro 01).

Quadro 1 - Conversão do valor nominal (gF) de cada MWS para log10. Modificado de Cardoso et $\mathrm{al}^{16}$ e Sorri ${ }^{15}$

\begin{tabular}{|l|r|r|r|l|}
\hline COR & \multicolumn{1}{|l|}{ gF } & \multicolumn{1}{l|}{ x100 } & \multicolumn{1}{l|}{ LOG 10 } & INTERPRETAÇÃO x COR DO MONOFILAMENTO \\
\hline VERDE & 0,05 & 5 & 0,6990 & Sensibilidade normal. \\
\hline AZUL & 0,2 & 20 & 1,3010 & Sensibilidade diminuída, mas ainda na faixa de normalidade. \\
\hline VIOLETA & 2,0 & 200 & 2,3010 & Sensibilidade protetora diminuída. \\
\hline VERMELHO & 4,0 & 400 & 2,6021 & Perda da sensação protetora da mão. \\
\hline LARANJA & 10,0 & 1000 & 3,0000 & Perda da sensação protetora do pé. \\
\hline ROSA & 300,0 & 30000 & 4,4771 & Sensibilidade residual à pressão profunda e dor. \\
\hline PRETO & 900,0 & 90000 & 4,9542 & Perda da sensibilidade a pressão profunda. \\
\hline
\end{tabular}

Fonte: Própria da autoria. 


\subsection{Análise dos dados e testes estatísticos}

Os dados foram ordenados em planilhas específicas por grupo e alinhados de acordo com os tempos de checagem. Para o GCS, o agrupamento foi feito para a mão direita e mão esquerda. Quando necessário, os dados foram aglutinados para obtenção de média e o desvio padrão desse grupo. Já, no agrupamento GTA as mãos foram tratadas separadamente: acometida (contralesional) e sã (ipsilesional).

Nos testes estatísticos, foram aplicados a Análise de Variância (ANOVA), pós-teste de Bonferroni, e o Teste t de Student, nos grupos. O índice de significância para valores de $\mathrm{p}<0,05$. Ao final, entre os registros de estesiometria (contralesional) e da MIF, apenas do GTA, foi estabelecida a Análise de Correlação para se identificar se houve consonância em resposta ao tratamento e validar o artifício de conversão logaritmo como representativa da evolução dos pacientes. Para isso, aplicou-se a correlação não-paramétrica de Spearman, intervalo de confiança (IC) de 95\% e significância estatística estabelecida para $p<0,05$ e coeficiente de correlação (r). Todas as análises estatísticas e a construção gráfica em coluna, média e desviopadrão (média \pm DP), foram realizadas no programa GraphPad Prism 6.0.

\section{Resultados}

\subsection{Os valores logarítmicos são coerentes e representativos da percepção tátil}

A partir dos resultados do GCS, observa-se a homogeneidade da percepção sensitiva tátil entre as mãos e a validação da consistência do protocolo da coleta dos dados. Contudo, os gráficos evidenciam também a disparidade das informações aferidas entre os valores nominais frente a conversão logarítmica (Fig. 2). De um lado, é apontado o resultado para o monofilamento azul (Fig. 2A) e, do outro, para o verde (Fig. 2B). Esta diferença qualitativa entre os gráficos é sustentada pela discrepância numérica dos valores nominais (gF) comparado aos valores convertidos, em logaritmo, que tornam esta última notação numérica mais condizente com o estado físico e funcional dos voluntários saudáveis. 
Figura 2 - Exposição dos resultados da estesiometria do GCS
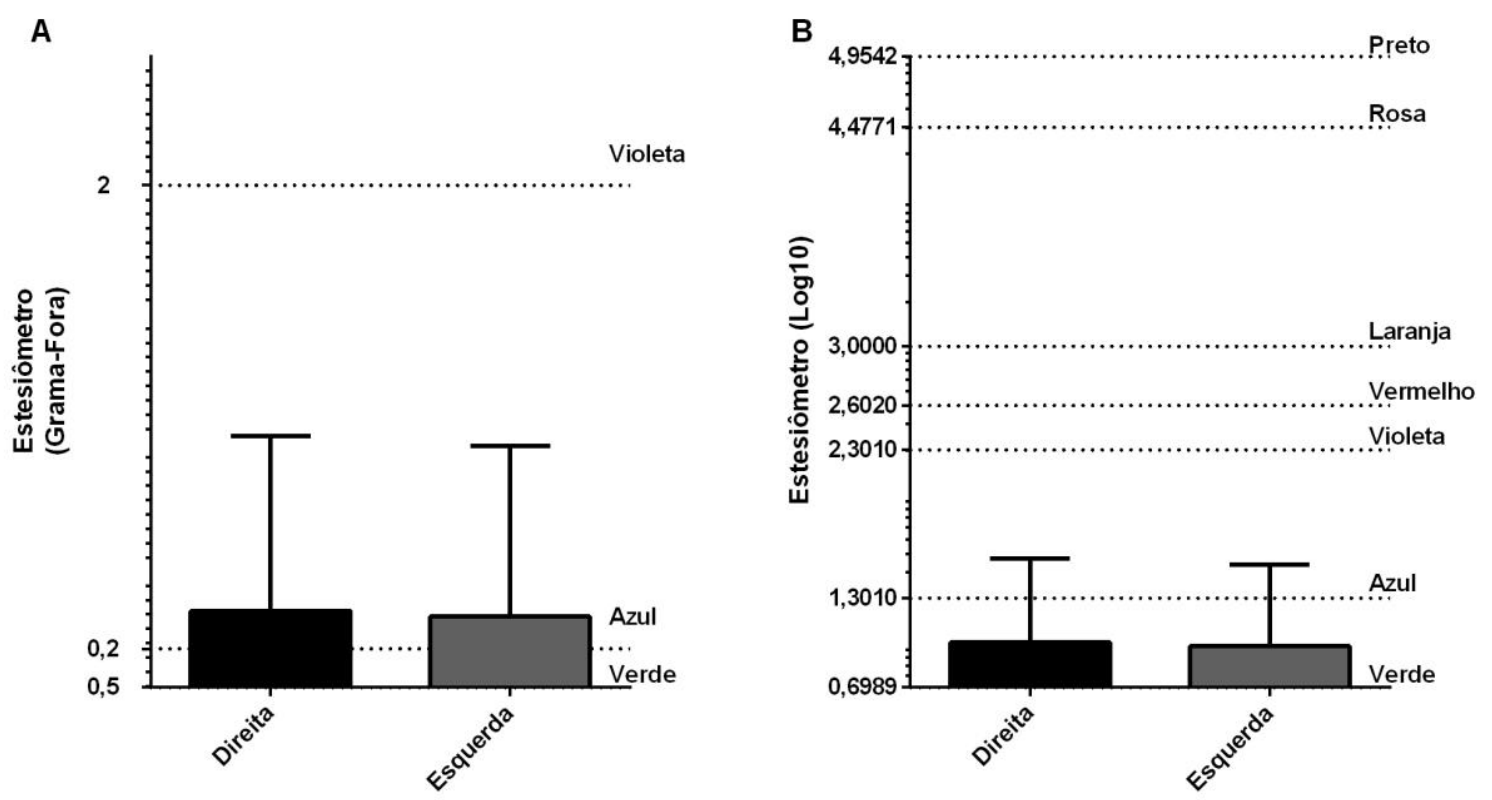

Legenda: A. Gráfico em valor nominal, grama-força (no eixo y) - Escala de 0,05 a 2,5 (gF). B. Gráfico em valores logarítmicos de base 10 (no eixo y). À esquerda de cada gráfico está a apresentação descritiva das cores dos monofilamentos.

Fonte: Própria da autoria.

\subsection{Os valores nominais não geraram inferências como na conversão logarítmica}

Ao estender o achado do GCS à interpretação do estado físico do GTA, percebe-se que os comportamentos dos desenhos gráficos são semelhantes, resguardada as respectivas proporções entre as escalas usadas (Fig 3). Como no resultado anterior, a figura 3 demonstra divergências qualitativas na percepção tátil no GTA. Na admissão, se a análise fosse feita apenas a partir de valores nominais, a conclusão estatística demonstraria significativo prejuízo à sensibilidade da mão acometida $(274,8 \pm 325,4$ e 33,6 $\pm 87,6$ - p<0,05), ainda que estivesse representada pelo monofilamento violeta (Fig. 03 A e B). Na alta, estatisticamente esse prejuízo não se manteria, apesar do grande distanciamento numérico $(49,2 \pm 137,4$ e $0,49 \pm 0,71-p>0,05)$ e qualitativo entre as mãos, respectivamente, os monofilamentos violeta e azul (Fig. 3 A e B). Esses resultados, mesmos aparentemente incoerentes entre si, levariam o avaliador a inferir pela recuperação funcional do grupo (Fig. 3 A e B). 
Figura 3 - Apresentação do GTA nos tempos de avaliação de admissão ( ${ }^{\text {a }}$ sessão) e alta $\left(20^{\mathrm{a}}\right.$ sessão)

A

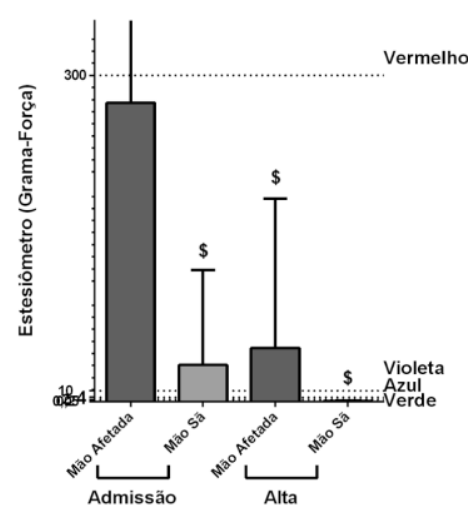

B

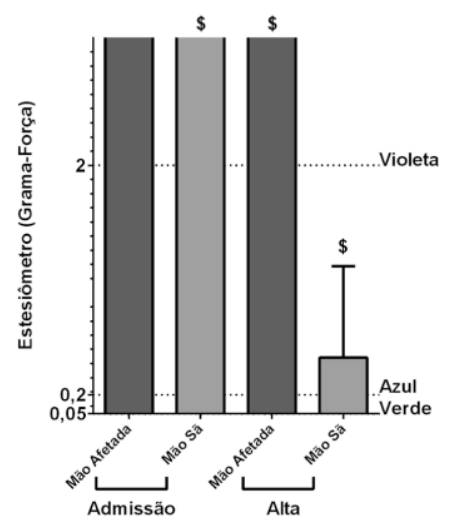

C

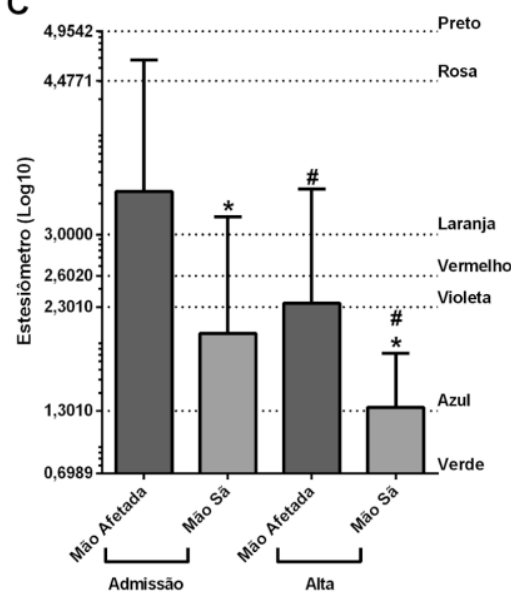

Legenda: Mão afetada (cinza escuro) e mão sã (cinza claro). A. Ordenamento pelo valor nominal, grama-força, em escala de 0,05 a $350(\mathrm{gF})$. B. Magnificação do trecho não legível do gráfico A, abaixo da linha tracejada do monofilamento violeta - escala de 0,05 a 3,0 (gF). C. Gráfico construído a partir da conversão logarítmica dos valores nominais. As linhas tracejadas esboçam os limites perceptivos e a cor descritiva correspondente de cada monofilamento, à esquerda de cada gráfico. Símbolos, significância estatística ( $<<0,05)$ : Cifrão $(\$)$, comparação com a coluna da mão afetada na admissão; Asterisco (*), comparação com a coluna da mão afetada no respectivo tempo de avaliação; e hashtag (\#), comparação com a coluna mão afetada na admissão

Fonte: Própria da autoria.

De modo diferente, os resultados da conversão logarítmica versam outra lógica interpretativa àquela observada pelos valores nominais (Fig. 03C). Na admissão, a mão afetada evidencia seu estado de prejuízo funcional para a percepção tátil, cuja média no GTA leva ao registro no monofilamento laranja, ao passo que a mão sã, como esperado, manifesta algum grau de prejuízo (monofilamento azul), mas com notação de normalidade (Quadro 01). Esta inferência é respaldada pela estatística $(3,4 \pm 1,2$ e 2,0 $\pm 1,1-\mathrm{p}<0,05)$. Na alta, a mão afetada mostra evolução para o monofilamento violeta $(2,3 \pm 1,0)$ e a mão sã apresenta tendência para sair da projeção do monofilamento azul $(1,3 \pm 0,5)$. A análise desses resultados, possibilita a conclusão que ao final do tratamento, o GTA evidencia melhora moderada na percepção tátil, sem alcançar o estado de normalidade da mão sã ( $p>0,05)$. Esta inferência é diferente daquela extraída da análise estatística dos valores nominais, descrita anteriormente.

\subsection{A conversão logarítmica é representativa e demonstra a evolução ao tratamento}

Os gráficos da figura 04 corroboram com os achados anteriores. O desenho dos gráficos pelas análises nominal e logarítmica é, consistentemente, o mesmo (Fig. 4). Contudo, a evolução do GTA comparado ao GCS, a partir dos dados de valores nominais dos monofilamentos, conduz à inferência que, a mão afetada na alta do GTA, a percepção da 
sensibilidade tátil melhora substancialmente, mas sem diferença estatística do GCS $(0,3 \pm 0,6$ e $49,2 \pm 137,4-p>0,05)$. Isso parece incoerente se observada as cores dos monofilamentos ao final, respectivamente, o violeta e o verde (Fig. 4 A e B).

Figura 4 - Apresentação comparativa da média das mãos do GCS com a mão afetada do GTA nos três tempos de avaliação

A

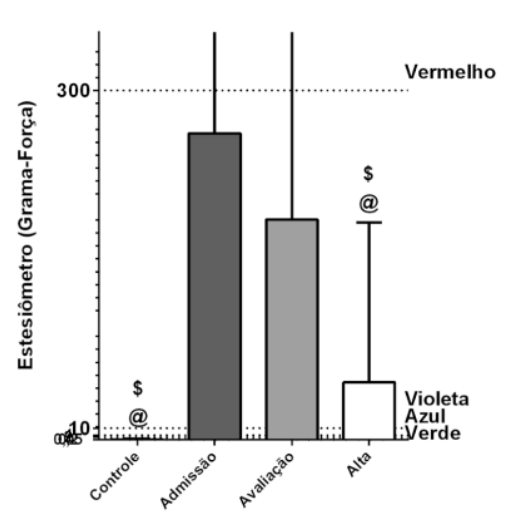

B

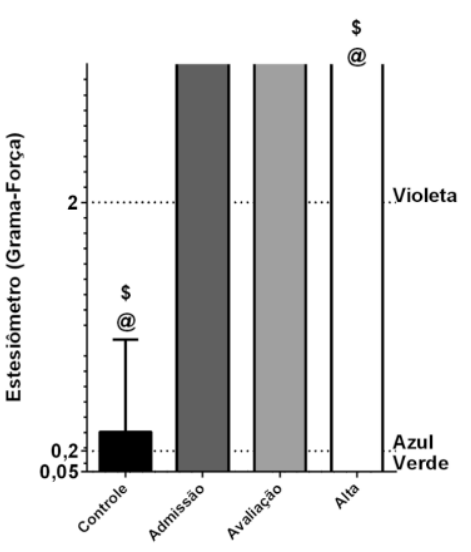

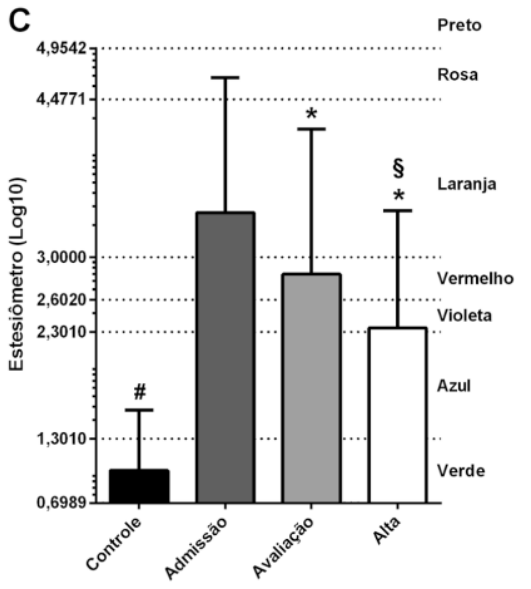

Legenda: GCS (preto), Mão afetada na: Admissão (cinza escuro), Avaliação (cinza claro) e alta (branco). A. Ordenamento pelo valor nominal, grama-força, em escala de 0,05 a 350 (gF). B. Magnificação do trecho não legível do gráfico A, abaixo da linha tracejada do monofilamento violeta - escala de 0,05 a 3,0 (gF). C. Gráfico construído a partir da conversão logarítmica dos valores nominais. As linhas tracejadas esboçam os limites perceptivos a cor descritiva correspondente de cada monofilamento, à esquerda de cada gráfico. Símbolos, significância estatística ( $\mathrm{p}<0,05)$ : Arroba (@), comparação da coluna do controle com as colunas de mão afetada de admissão e alta. Cifrão (\$), comparação com a coluna da mão afetada na alta com as colunas de admissão e avaliação; hashtag (\#), comparação da coluna controle com as três colunas de avaliação mão afetada; Asterisco (*), comparação com a coluna de admissão da mão afetada; e Signum Sectionis (§), comparação da coluna de alta da mão afetada com a coluna de avaliação

Fonte: Própria da autoria.

Mais consistente com esse achado descritivo das cores dos monofilamentos, ao final do tratamento comparado com o controle, é a conversão logarítmica, que consegue expressar a

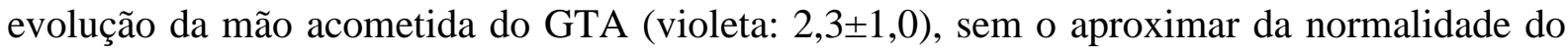
GCS (verde: $1,0 \pm 0,5$ ), que são estatisticamente diferentes ( $p>0,05$ ), figura 04C.

\subsection{A correlação da mif com a estesiometria confirma a recuperação do gta e a uniformidade da conversão em log 10}

Os achados da estesiomentria e da MIF do GTA foram correlacionados. Tanto os valores nominais $(\mathrm{gF})$ quanto a conversão em Log10 dos monofilamentos do estesiômetro demonstram a recuperação dos pacientes (Fig. 5). Pelos valores nominais (Fig. 5A), mesmo com dados sobrepostos, há significância estatística dos resultados da estesiometria e evidencia a recuperação funcional e física dos pacientes ( $p=0,026$; IC: $-0,87$ a -0,08; e r= -0,61). Os dados convertidos dos monofilamentos demonstram capacidade interpretativa condizente com os 
valores nominais (Fig. 5B), e ainda manifestam distribuição mais harmônica sem provocar alteração da interpretação final ( $p=0,030$; IC: -0,86 a -0,05; e r= -0,59). Disso, infere-se que a conversão em Log10 não modifica a tendência dos resultados e realça a uniformização dos dados.

Figura 5 - Correlação MIF x estesiometria
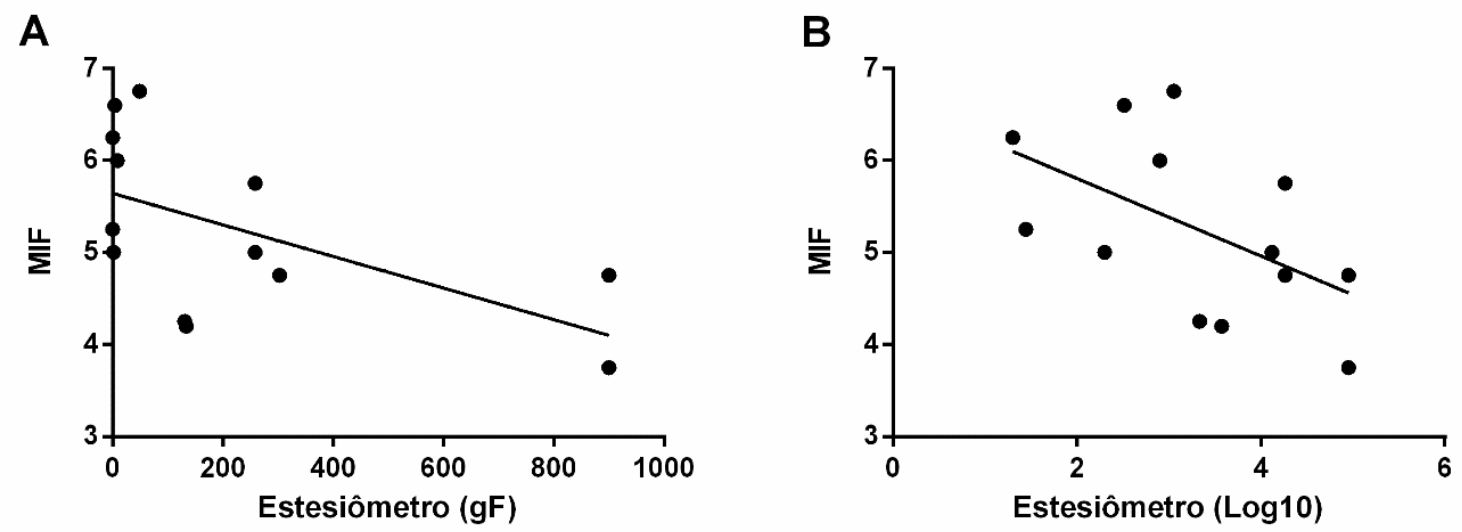

Legenda: Comportamento evolutivo dos pacientes GTA em resposta ao tratamento, em função das três janelas temporais de checagem. A. valor nominal $(\mathrm{gF})$. B. Conversão $(\log 10)$.

Fonte: Própria da autoria.

\section{Discussão}

Os resultados sugerem que a análise numérica dos dados dos monofilamentos do estesiômetro consegue representar a evolução do estado físico quanto à percepção da sensibilidade tátil dos grupos, GCS e GTA. Entre as opções aqui comparadas, a conversão em logaritmo demonstra sensibilidade, coerência e consistência dos dados, expondo qualidade e acurácia maior que o seu paralelo, por valores nominais $(\mathrm{gF})$ dos monofilamentos. Além disso, a conversão aplicada garante a reprodutividade da análise, tanto na condição de grupo controle como de tratamento. Ressalta-se que a representação numérica em logaritmo não reduz a importância do equivalente qualitativo, pelas cores dos monofilamentos, e não altera a tendência do resultado final, mas garante a uniformização dos achados e possibilita interpretações mais ricas.

A busca pela objetividade em pesquisa, a partir dos dados de estesiometria, não é recente. Classicamente, pelo menos desde o início do século, pesquisadores já se valiam de notações numéricas para gerar inferência dos resultados, exemplo disso, é a avaliação da sensibilidade da área pós-cirúrgica por técnicas de mamoplastia, usando o conjunto com 20 monofilamentos de Semmes-Weinstein ${ }^{17}$, isso aumenta a acurácia entre as unidades do 
estesiômetro. Esse resultado é apresentado em massa por área $\left(\mathrm{g} / \mathrm{mm}^{2}\right)$, sem qualquer conversão. Esta notação é semelhante àquela do estesiômetro produzido pela Sorri-Bauru ${ }^{\circledR}$, grama-força. Essa hipótese é reforçada por um estudo de revisão mais recente que, no conjunto com 20 monofilamentos, as discrepâncias são menores, entre os valores nominais ${ }^{18}$. Achado semelhante foi produzido com o uso de monofilamentos de von Frey, no qual os dados demonstrados foram mais próximos, com menores desvios ${ }^{19}$. Isso respalda a ideia aqui apresentada de conversão logarítmica, para se alcançar a congruência dos dados, na impossibilidade do uso de conjunto de estesiômetro com vários monofilamentos.

Reforça o argumento de conversão em logaritmo, o entendimento que a calibração do conjunto de monofilamentos de von Frey segue uma escala logarítmica de 0,08 a $2.943 \mathrm{mN}$ $(0,008 \mathrm{~g}-300 \mathrm{~g})^{20}$. Trata-se de um achado comum entre os dispositivos e justifica a coerência de dados apresentados em outros estudos ${ }^{19,21}$. Tal coerência, contudo, não parece ser mantida em conjuntos de estesiômetro com menor quantidade de monofilamentos disponíveis, como os aqui convertidos. Assim, entende-se que o princípio da escala logarítmica é a base de confecção dos monofilamentos, e respalda a conversão em logaritmo executada nessa proposta. Esse procedimento é sustentado pela Lei de Weber-Fechner ${ }^{22}$, sintetizada da seguinte forma: Weber (1846) considerava que a percepção de um estímulo estava relacionada à força inicialmente exercida; já Fechner (1860) definiu que a percepção ao estímulo seguia uma escala logarítmica - esta é a notação científica aplicada aos monofilamentos ${ }^{22}$.

Outras metodologias de conversão numérica dos monofilamentos já foram abordadas por padronização em escala de números inteiros, de 0 a 5 , onde, respectivamente, o zero indicando a ausência de resposta sensitiva e, o cinco, a sensibilidade ao dispositivo mais delicado $^{23}$. Esta padronização, em substituição aos valores nominais dos monofilamentos, reduziu as discrepâncias numéricas e se assemelha à justificativa que sustenta os procedimentos aqui apresentados, de conversão logarítmica.

Sobre isso, reconhece-se que o manual do estesiômetro Sorri-Bauru ${ }^{\circledR}$ também apresenta formulação matemática por pressão $(\mathrm{gF})$, em escala linear, baseada em logaritmo (por dez vezes a força aplicada, em miligramas $)^{15}$. Esta equação pode ser objeto de estudos futuros. Igualmente, ao estabelecimento de validação e confiabilidade dos resultados desta proposta, por comparações dos valores nominais e logarítmicos a partir de análise de correlação com a estesiometria digital. Acredita-se que esse método tende a consolidar as afirmativas aqui apresentadas.

À luz do principal resultado, que evidencia a conversão logarítmica como uma eficiente opção de análise dos dados da avaliação estesiométrica, o presente estudo manifesta limitações, 
entre as quais são destacados três pontos. Primeiro, apesar de a amostra ser homogênea entre tratados e controles, não houve desequilíbrio entre as faixas etárias, em prejuízo aos mais jovens, uma vez que a maior parte dos grupos foram compostos por pessoas mais velhas. Reconhecidamente, diferentes sistemas corporais declinam com a idade e possibilitam o surgimento de alterações físico funcionais, como a disfunção nervosa periférica e complicações $\operatorname{associadas}^{24}$. Por isso, acredita-se que os resultados evolutivos apresentados poderiam ser mais acentuados. Outros estudos devem ser feitos com amostras mais jovens para afastar esta possibilidade de viés levantada.

Segundo os pacientes do grupo tratado eram portadores de sequelas de AVE, uma doença neurológica aguda focal, de localização não descrita nos laudos recebidos dos pacientes do grupo tratado - o diagnóstico foi mais clínico do que subsidiado por exames de imagem. Essa situação se caracteriza como limitante pela incerteza do território vascular acometido, se era mais motor ou sensitivo; se era mais associativo ou de projeção. Por esta razão, optou-se pela nomenclatura de percepção (da sensibilidade) tátil nesse estudo ${ }^{25}$, e não apenas sensibilidade tátil, já que o prejuízo base dos pacientes era central, ao passo que as vias periféricas se encontravam íntegras.

E, terceiro, além do exposto, o objeto de registro dos monofilamentos do estesiômetro foram fundamentados nas neuropatias periféricas ${ }^{26}$, mas também vem se mostrando um instrumento de avaliação tátil confiável e aplicável em estudos com doenças neurológicas centrais $^{27,28}$, em particular o $\mathrm{AVE}^{25}$. No presente estudo, fez-se a correlação da resposta perceptiva tátil pela recuperação funcional. Por serem escalas com pontuação de interpretação inversa, a correlação foi previsivelmente negativa e representou a evolução dos pacientes em função do tratamento. Sobre isso, acredita-se que a magnitude desse achado pode ser aumentada se a comparação for realizada com outro teste de sensibilidade validado, como o Teste de Discriminação de Dois-Pontos, também amplamente usado em pesquisa com pacientes com sequelas cerebrovasculares ${ }^{29}$.

\section{Conclusão}

Conclui-se que o procedimento proposto, em logaritmo de base 10, para o estesiômetro Sorri-Bauru $^{\circledR}$, pode ser tratado como uma opção de análise numérica com excelente poder de representatividade, viabilidade e reprodutividade que fornece objetividade aos dados, principalmente para a pesquisa em reabilitação e facultado às áreas clínicas e práticas. Justamente por esse último motivo, não é indicado o abandono das referências qualitativas dos 
monofilamentos e sim, a associação do resultado logarítmico com a notação descritiva da cor do monofilamento correspondente, para melhor representar o estado de sensibilidade tátil em acompanhamento. Outros estudos devem ser realizados focando na validação com diferentes faixas etárias e acometimentos físicos e funcionais.

\section{Referências}

1. Fonseca MDCR, Elui VMC, Lalone E, Da Silva NC, Barbosa RI, Marcolino AM, et al. Functional, motor, and sensory assessment instruments upon nerve repair in adult hands: Systematic review of psychometric properties. Syst Rev. 2018 Oct 27;7(1):175.

2. Rinderknecht MD, Dueñas JA, Held JP, Lambercy O, Conti FM, Zizlsperger L, et al. Automated and Quantitative Assessment of Tactile Mislocalization After Stroke. Front Neurol. 2019 Jun 12;10:593.

3. Kessner SS, Bingel U, Thomalla G. Somatosensory deficits after stroke: a scoping review. Top Stroke Rehabil. 2016 Apr;23(2):136-46. Epub 2016 Jan 13.

4. Suzuki M, Sugimura S, Suzuki T, Sasaki S, Abe N, Tokito T, et al. Machine-learning prediction of self-care activity by grip strengths of both hands in poststroke hemiplegia. Medicine. 2020 Mar 13;99(11):e19512.

5. Van De Ven-Stevens LA, Graff MJ, Selles RW, Schreuders TA, Van Der Linde H, Spauwen PH, et al. Instruments for assessment of impairments and activity limitations in patients with hand conditions: A European Delphi study. J Rehabil Med. 2015

Nov;47(10):948-56.

6. Escalona-Marfil C, Coda A, Ruiz-Moreno J, Riu-Gispert LM, Gironès X. Validation of an electronic visual analog scale mHealth tool for acute pain assessment: Prospective crosssectional study. J Med Internet Res. 2020 Feb 12;22(2):e13468.

7. Adami C, Lardone E, Monticelli P. Inter-rater and inter-device reliability of mechanical thresholds measurement with the Electronic von Frey Anaesthesiometer and the SMALGO in healthy cats. J Feline Med Surg. 2019 Oct;21(10):979-984. Epub 2018 Nov 22.

8. Sapa MC, Sagot JC, Touvenot G, Xavier F, Hidalgo Diaz JJ, Facca S, et al. Comparison of tactile sensitivity measured with a new digital esthesiometer (Beam Test) relative to SemmesWeinstein monofilament analog esthesiometer. Hand Surg Rehabil. 2019 Sep;38(4):242-245. Epub 2019 Jun 26.

9. Chikai M, Ozawa E, Takahashi N, Nunokawa K, Ino S. Evaluation of the variation in sensory test results using Semmes-Weinstein monofilaments. Annu Int Conf IEEE Eng Med Biol Soc. 2015 Aug;2015:1259-62.

10. Abraira VE, Ginty DD. The sensory neurons of touch. Neuron. 2013 Aug 21;79(4):61839. 
11. Siqueira GSA, Hagemann PMS, Coelho DS, Santos FHD, Bertolucci PHF. Can MoCA and MMSE Be Interchangeable Cognitive Screening Tools? A Systematic Review. Gerontologist. 2019 Nov 16;59(6):e743-e763.

12. Cavaco NS, Alouche SR. Instrumentos de avaliação da função de membros superiores após acidente vascular encefálico: uma revisão sistemática. Fisioter e Pesqui. 2010 AbrJun;17(2):178-183.

13. Riberto M, Miyazaki MH, Jucá SSH, Sakamoto H, Pinto PPN, Battistella, LR. Validação da Versão Brasileira da Medida de Independência Funcional Validation of the Brazilian version of Functional Independence Measure. Acta Fisiatr. 2004 Ago;11(2):72-76.

14. Marcolino AM, Barbosa RI, Souza DNA de, Rebelo R de B, Delgado PM, Mazzer N, et al. Correlation between Moberg Pick-Up test and sensation threshold test after median nerve reconstruction. Acta Fisiatr. 2012 Dec 05;19(4):216-21.

15. SORRI. Sociedade de Reabilitação e Reintegração do Incapacitado. Estesiômetro Sorri: kit para teste de sensibilidade cutânea - Manual do usuário [Internet]. Bauru: Sorri; 2020. [Acesso em 10 abril 2020]. Disponível em: https://sorribauru.com.br/custom/678/uploads/manual_kit_portugues.pdf.

16. Cardoso MM, Lobo DC, Cruz MJD, Monteiro RPA, Seabra AD, Paixão GMD, et al. Abordagens específicas em Terapia Ocupacional em reabilitação após Acidente Vascular Encefálico. Rev Interinst Bras Ter Ocup. 2019 Abr 30;3(2):191-209.

17. Hamdi M, Greuse M, DeMey A, Webster MH. A prospective quantitative comparison of breast sensation after superior and inferior pedicle mammaplasty. Br J Plast Surg. 2001 Jan;54(1):39-42.

18. Antonarakis GS, Christou P. Quantitative evaluation of neurosensory disturbance after bilateral sagittal split osteotomy using semmes-weinstein monofilaments: A systematic review. J Oral Maxillofac Surg. 2012 Dec;70(12):2752-60. Epub 2012 Sep 23.

19. Roldan CJ, Johnson C, Lee SO, Peng A, Dougherty PM, Huh B. Subclinical peripheral neuropathy in patients with head and neck cancer: A quantitative sensory testing (QST) study. Pain Physician. 2018 Jul;21(4):e419-e427.

20. Tena B, Escobar B, Arguis MJ, Cantero C, Rios J, Gomar C. Reproducibility of electronic von Frey and von Frey monofilaments testing. Clin J Pain. 2012 May;28(4):318-23.

21. Kuo LC, Yang CJ, Lin CF, Jou IM, Yang YC, Yeh CH, et al. Effects of a task-based biofeedback training program on improving sensorimotor function in neuropathic hands in diabetic patients: A randomized controlled trial. Eur J Phys Rehabil Med. 2019 Oct;55(5):618-626. Epub 2019 May 3.

22. Bradman MJG, Ferrini F, Salio C, Merighi A. Practical mechanical threshold estimation in rodents using von Frey hairs/Semmes-Weinstein monofilaments: Towards a rational method. J Neurosci Methods. 2015 Nov 30;255:92-103. Epub 2015 Aug 18. 
23. Schreuders TA, Selles RW, van Ginneken BT, Janssen WG, Stam HJ. Sensory Evaluation of the Hands in Patients with Charcot-Marie-Tooth Disease Using Semmes-Weinstein Monofilaments. J Hand Ther. 2008 Jan-Mar;21(1):28-34.

24. Carrer P, Trevisan C, Curreri C, Giantin V, Maggi S, Crepaldi G, et al. SemmesWeinstein Monofilament Examination for Predicting Physical Performance and the Risk of Falls in Older People: Results of the Pro.V.A. Longitudinal Study. Arch Phys Med Rehabil. 2018 Jan; 99(1):137-143. Epub 2017 Sep 20.

25. Dias C da S, Alferi FM, Battistella LR. Utilização de monofilamentos para avaliação sensorial em pacientes com sequela de Acidente Vascular Encefálico (AVE) - uma revisão sistemática. Rev bras neurol. 2019 Oct; 55(3):22-8.

26. Williams D, Conn J, Talley N, Attia J. Reviewing the evidence base for the peripheral sensory examination. Int J Clin Pract. 2014 Jun; 68(6):756-60.

27. Ofek H, Alperin M, Knoll T, Livne D, Laufer Y. Assessment of texture discrimination ability at the sole of the foot in subjects with chronic stroke compared with young and elderly subjects with no neurological deficits: a reliability and validity study. Disabil Rehabil. 2018 Aug; 40(16):1960-1966. Epub 2017 May 8.

28. Ellaway PH, Catley M. Reliability of the electrical perceptual threshold and SemmesWeinstein monofilament tests of cutaneous sensibility. Spinal Cord. 2013 Feb; 51(2):120-5. Epub 2012 Sep 4.

29. Arya KN, Pandian S, Vikas, Puri V. Mirror Illusion for Sensori-Motor Training in Stroke: A Randomized Controlled Trial. J Stroke Cerebrovasc Dis. 2018 Nov; 27(11):3236-3246. Epub 2018 Aug 16. 